\title{
Recrutamento de médicos cubanos: análise comparativa da estratégia adotada por Angola, Portugal e Brasil para compor seus recursos humanos em saúde
}

Recruitment of Cuban doctors: comparative analysis of the strategy adopted by Angola, Portugal and Brazil to compose their human resources in health

Reclutamiento de médicos cubanos: análisis comparativo de la estrategia adoptada por Angola, Portugal y Brasil para componer sus recursos humanos en salud

\author{
Sandra Mara Campos Alves ${ }^{1,2}$ \\ Maria Célia Delduque ${ }^{3}$ \\ Paula Lobato de Faria ${ }^{4}$
}

\section{Resumo}

O provimento de médicos em quantidade adequada e distribuídos de forma equilibrada pelo território é um dos grandes obstáculos ao acesso da população a ações e serviços de saúde, o que tem conduzido a processos de recrutamento internacional pelos países com déficit. Este estudo de caso múltiplo comparou as estratégias jurídico-institucionais utilizadas por Angola, Brasil e Portugal para a efetivação do recrutamento de médicos cubanos e os benefícios gerados aos países a partir dessa interação. Foram previamente selecionados os aspectos gerais do acordo de cooperação; os aspectos legais para o exercício da medicina por estrangeiros; e os benefícios gerados a partir da cooperação firmada. Foram realizadas análise dos acordos de cooperação firmados entre os países investigados e entrevista de 30 pessoas, divididas em quatro subgrupos, compostos por meio da amostragem bola de neve. Os resultados apontam que estratégias utilizadas pelos países envolvidos na cooperação médica por vezes se aproximam, mas contextos específicos conduzem à busca por soluções peculiares, que se harmonizem com a legislação vigente em cada país. Conclui-se pelo necessário fortalecimento do suporte jurídico voltado a apoiar o agir estatal na área da cooperação em saúde, visto que o ambiente de governança é complexo e dinâmico.

\section{Palavras-chave}

Recursos Humanos em Saúde. Cooperação internacional. Acesso Universal aos Serviços de Saúde.

\begin{abstract}
The provision of doctors in an adequate quantity and distributed in a balanced way across the territory is one of the major obstacles to the population's access to health actions and services, which has led to international recruitment processes by countries with deficits. This multiple

\footnotetext{
${ }^{1}$ Este artigo é resultado de pesquisa de doutorado apoiada pelo CAPES, por meio de Programa de Doutorado Sanduíche (PDSE processo n 8881.132979/2016-01).

2 Doutora em Saúde Coletiva, Universidade de Brasília, Brasília, DF, Brasil; Coordenadora, Programa de Direito Sanitário, Fundação Oswaldo Cruz, Brasília, DF, Brasil. https://orcid.org/0000-0002-3615-0225. E-mail: smcalves@gmail.com

${ }^{3}$ Doutora em Saúde Pública, Universidade de São Paulo, São Paulo, SP, Brasil; Professora, Departamento de Saúde Coletiva, Universidade de Brasília, Brasília, DF, Brasil. https://orcid.org/0000-0002-5351-3534. E-mail: mcdelduque@gmail.com

${ }^{4}$ Doutora em Direito Público, Université Montesquieu, Bordeaux, França; Professora Associada, Departamento de Ciências Sociais em Saúde, Escola Nacional de Saúde Pública, Universidade Nova de Lisboa, Lisboa, Portugal; Membro da CICS.NOVA e CISP/ENSP, Universidade Nova de Lisboa, Lisboa, Portugal. https://orcid.org/0000-0002-3290-4480. E-mail: pa.lobfaria@ensp.unl.pt
} 
case study compared the legal-institutional strategies used by Angola, Brazil and Portugal to effectively recruit Cuban doctors, and the benefits generated to the countries from this interaction. The dimensions of general aspects of the cooperation agreement were previously selected; legal aspects for the practice of medicine by foreigners and the benefits generated from the cooperation signed. An analysis of the cooperation agreements signed between the investigated countries was carried out and 30 people were interviewed, divided into 4 subgroups, composed by snowball sampling. The results show that the strategies used by the countries involved in medical cooperation are sometimes close, but specific contexts lead to the search for peculiar solutions that harmonize with the legislation in force in each country. It is concluded that there is a need to strengthen legal support aimed at supporting state action in cooperation in health, since the governance environment is complex and dynamic.

\section{Keywords}

Health Workforce. International Cooperation. Universal Access to Health Care Services.

\section{Resumen}

La provisión de médicos en un número adecuado y distribuidos de manera equilibrada en el territorio es uno de los mayores obstáculos para el acceso de la población a las acciones y servicios de salud, lo que ha llevado a procesos de contratación internacional por países con déficit. Este estudio de caso múltiple comparó las estrategias jurídico-institucionales utilizadas por Angola, Brasil y Portugal para reclutar médicos cubanos, y los beneficios que genera a los países esta interacción. Previamente se seleccionaron las dimensiones de los aspectos generales del convenio de cooperación; Aspectos legales para el ejercicio de la medicina por parte de extranjeros y los beneficios generados por la cooperación firmada. Se realizó un análisis de los convenios de cooperación firmados entre los países investigados y se entrevistó a 30 personas, divididas en 4 subgrupos, compuestos por muestreo de bola de nieve. Los resultados muestran que las estrategias utilizadas por los países involucrados en la cooperación médica son en ocasiones cercanas, pero contextos específicos llevan a la búsqueda de soluciones peculiares que armonicen con la legislación vigente en cada país. Se concluye que el necesario fortalecimiento de los apoyos legales orientados a apoyar la acción estatal en el área de cooperación en salud, ya que el entorno de gobernanza es complejo y dinámico.

\section{Palabras clave}

Fuerza Laboral en Salud. Cooperación Internacional. Acceso Universal a los Servicios de Salud.

Prover recursos humanos em saúde (RHS), especialmente médicos, em quantidade adequada e distribuídos de forma equilibrada pelo território, é um dos grandes desafios enfrentados pelos Estados, independente do modelo de sistema de saúde adotado, ou ainda de suas condições socioeconômicas (1-3). Dados da Organização Mundial da Saúde (OMS) (4) demonstram a disparidade entre a concentração de regiões com carga global de doenças e o número de trabalhadores de saúde.

O recrutamento de RHS provenientes de outros países tem sido utilizado como estratégia para mitigar essa escassez e reforçar o quadro de trabalhadores, especialmente no âmbito da atenção primária em saúde (APS), sendo inclusive uma das metas dos Objetivos de Desenvolvimento Sustentável defendidos pela Organização das Nações Unidas 
(ONU). Angola, Portugal e Brasil, países lusófonos com características sociodemográficasepidemiológicas distintas, recrutaram médicos cubanos para suprimir seu déficit e ampliar o acesso da população a ações e serviços de saúde.

A República de Cuba, com uma densidade de 7,5 médicos por 1.000 habitantes (5), é internacionalmente reconhecida por sua política de formação na área de recursos humanos em saúde, com destaque para a medicina geral e integral, que propiciou, ao longo dos anos, a apresentação de indicadores de saúde que a comparam a países desenvolvidos (6-7). Possui ações de cooperação médica em mais de 60 países (5).

O artigo teve como objetivo comparar as estratégias jurídico-institucionais utilizados por Angola, Brasil e Portugal para a efetivação do recrutamento de médicos cubanos e os benefícios gerados aos países a partir dessa interação.

\section{Metodologia}

Trata-se de estudo de caso múltiplo qualitativo-descritivo-analítico, com triangulação de dados a partir de coleta de informações em fontes múltiplas de evidência (documentos e entrevistas focadas).

As evidências coletadas visaram trazer subsídios para comparar a cooperação estabelecida com Cuba, nas seguintes dimensões: i) aspectos gerais do acordo de cooperação (tipo de cooperação, obrigações assumidas; contraprestação pelos serviços prestados; etc.); ii) aspectos legais para o exercício da medicina por estrangeiros; iii) benefícios gerados a partir da cooperação firmada.

Foram consultados os acordos de cooperação firmados e suas respectivas renovações; documentos técnicos; relatórios e memorando emitidos por órgãos governamentais, notadamente o Ministério da Saúde dos países investigados. Também foi realizada pesquisa legislativa sobre os sistemas de saúde e permissão para o exercício da medicina por estrangeiros.

Também foram realizadas 30 entrevistas focadas no período de junho/2017 a outubro/2018, em que se utilizou a amostragem bola de neve (snowball) (8-9) para a composição dos quatro subgrupos, de modo a congregar uma maior variedade de visões e opiniões sobre o tema.

A divisão dos subgrupos foi previamente estabelecida da seguinte forma: i) gestores de saúde de nível central: responsáveis pela tomada de decisão e monitoramento das ações relacionadas ao processo de recrutamento de médicos cubanos (ministros, secretários, diretores e coordenadores do Ministério da Saúde); ii) técnicos: profissionais responsáveis pelo acompanhamento, implementação e fiscalização das atividades exercidas pelos médicos 
cubanos e/ou pelo acompanhamento do acordo de cooperação; iii) médicos cubanos: médicos cubanos que trabalharam nos países investigados (Brasil, Angola e Portugal), ao abrigo dos acordos de cooperação; iv) outros: informantes que foram referenciados na cadeia de entrevistas e que não se encaixaram nas categorias anteriores, por exemplo, representantes da Ordem dos Médicos, médicos angolanos e médico espanhol (tabela 1).

Tabela 1. Perfil e quantitativo dos entrevistados, por país

\begin{tabular}{|c|c|c|c|c|}
\hline \multirow[t]{2}{*}{ Perfil } & \multicolumn{3}{|c|}{ País } & \multirow[t]{2}{*}{$\mathbf{n}$} \\
\hline & Portugal & Angola & Brasil & \\
\hline Gestores de saúde de nível central & 2 & 1 & 1 & 4 \\
\hline $\begin{array}{l}\text { Técnicos responsáveis pela condução e/ou acompanhamento do } \\
\text { processo de recrutamento de médicos cubanos }\end{array}$ & 3 & 1 & - & 4 \\
\hline $\begin{array}{l}\text { Médicos cubanos que trabalharam em Portugal; Brasil ou Angola, } \\
\text { cobertos pelos acordos de cooperação }\end{array}$ & 8 & - & 9 & 17 \\
\hline Outros & 3 & 2 & - & 5 \\
\hline TOTAL & 16 & 4 & 10 & 30 \\
\hline
\end{tabular}

Fonte: elaborada pelas autoras.

Os roteiros utilizados nas entrevistas foram distintos, respeitando a diversidade de respondentes e o conhecimento específico de cada um sobre o fenômeno analisado. As entrevistas foram realizadas no Brasil e em Portugal, presencialmente, e/ou por telefone, com o auxílio da chamada de voz do aplicativo WhatsApp.

A pesquisa foi aprovada no Comitê de Ética da Fiocruz Brasília (CAAE 58686316.7.0000.8027); na Comissão de Ética para a Saúde da ARSLVT de Portugal (1724/CES/2018) e Comitê de Ética do Ministério da Saúde de Angola (n³3/2017). O termo de consentimento livre e esclarecido (TCLE) foi obtido por escrito, antes do início de cada entrevista, e, quando autorizado, foi realizado o registro em áudio.

\section{Resultados}

Aspectos gerais

A comparação das características socioeconômico-demográficas de Angola, Portugal e Brasil evidenciam estágios distintos de desenvolvimento (tabela 2).

Tabela 2. Tabela comparativa das características socioeconômico-demográficas de Angola, Portugal e Brasil

\begin{tabular}{l|l|l|l}
\hline Características & \multicolumn{1}{|c}{ Angola } & \multicolumn{1}{c}{ Portugal } & \multicolumn{1}{c}{ Brasil } \\
\hline Área $(\mathrm{km} 2)$ & 1.246 .700 & 92.090 & $8.515 .767,049$ \\
\hline População (2017) & $29,310,272$ & $10,839,514$ & $207,353,392$ \\
\hline
\end{tabular}




\begin{tabular}{l|l|l|l}
\hline Características & \multicolumn{1}{c|}{ Angola } & \multicolumn{1}{c}{ Portugal } & \multicolumn{1}{c}{ Brasil } \\
\hline Ranking no IDH & $147^{\circ}(0,581)$ & $41^{\circ}(0,837)$ & $79^{\circ}(0,748)$ \\
\hline Índice de GINI & 42,7 & 35,5 & 51,3 \\
\hline PIB (bilhões \$) & 192 & 311,3 & 3,219 \\
\hline
\end{tabular}

Fonte: elaborada com base nas referências 10 e 11.

A diferença de condições de desenvolvimento entre os países reflete-se diretamente nas condições de saúde dos mesmos (tabela 3).

Tabela 3. Aspectos comparativos de condições de saúde de Brasil, Portugal, Angola e Cuba

\begin{tabular}{l|c|c|c}
\hline Países & $\begin{array}{c}\text { Taxa de mortalidade materna } \\
\text { (por 100.000 habitantes) }\end{array}$ & $\begin{array}{c}\text { Taxa de mortalidade } \\
\text { de menores de } \mathbf{5} \text { anos } \\
\text { (por } \mathbf{1 . 0 0 0} \text { habitantes) }\end{array}$ & $\begin{array}{c}\mathbf{N}^{\circ} \text { de médicos } \\
\text { (por } \mathbf{1 . 0 0 0} \\
\text { habitantes) }\end{array}$ \\
\hline Brasil & 44 & 15,1 & 1,9 \\
\hline Portugal & 10 & 3,5 & 4,4 \\
\hline Angola & 477 & 82,5 & 0,1 \\
\hline
\end{tabular}

Fonte: elaborado com base na referência 12.

Os acordos de cooperação celebrados entre Cuba e os países investigados apresentam características próprias, ajustadas às peculiaridades de cada país. (tabela 4)

Tabela 4. Informações sobre a cooperação cubana com Angola, Portugal e Brasil na área da saúde para a vinda de médicos cubanos

\begin{tabular}{l|c|c|c}
\hline Informações & Angola & Portugal & Brasil \\
\hline $\begin{array}{l}\text { Data da cooperação } \\
\text { com Cuba }\end{array}$ & Desde 29/07/1976 & Desde 13/06/2009 & Desde 22/08/2013 \\
\hline $\begin{array}{l}\text { Objetivo da } \\
\text { cooperação }\end{array}$ & $\begin{array}{l}\text { Cooperação } \\
\text { econômica e técnica- } \\
\text { científica }\end{array}$ & $\begin{array}{l}\text { Prover serviços médicos } \\
\text { em zonas geográficas } \\
\text { carenciadas }\end{array}$ & $\begin{array}{l}\text { Participação dos } \\
\text { médicos cubanos no } \\
\text { PMMB }\end{array}$ \\
\hline $\begin{array}{l}\text { Modalidade de } \\
\text { cooperação }\end{array}$ & ATC & ATC & $\begin{array}{c}\text { Triangulação } \\
\text { Brasil-OPAS-Cuba }\end{array}$ \\
\hline $\begin{array}{l}\text { No de médicos } \\
\text { cubanos nos países } \\
\text { recrutadores (2017) }\end{array}$ & 1.285 & 17 & 8.730 \\
\hline
\end{tabular}

Fonte: elaborada com base na referência 5 .

\section{Cooperação médica Angola-Cuba}

Data de 1976 o Acordo de Cooperação Econômica e Técnico-Científico firmado entre Angola e Cuba e sucessivos protocolos que regem a cooperação entre os países. A cooperação teve como objetivo a vinda de profissionais cubanos para atuar nos Ministérios 
da Saúde, Educação e Ensino Superior; serviços de saúde militares e serviços do Ministério do Interior (13). A modalidade de cooperação eleita por Cuba é a assistência técnica compensada (ATC), consistindo em um contrato direto com o país recrutador em que há contraprestação financeira calculada com base no número de profissionais enviados (14). No caso da ATC firmada com o Ministério da Saúde Angolano (MINSA), a representação do governo cubano é realizada por meio da Coorporação Antex S.A., sociedade mercantil de capital cubano que tem a sua disposição RHS para prestação de serviço na área da saúde (15).

O acordo firmado estabelece algumas obrigações para os estados contratantes. Ao governo cubano, incumbe a obrigação de enviar médicos com o perfil/qualificação solicitado, para prestar serviços pelo prazo de 12 meses, podendo haver renovação. Angola, além de responsável pelo pagamento direto pelos serviços prestados, também deveria garantir aos profissionais cubanos alojamentos mobiliados e climatizados, com internet; assumindo também os custos com os serviços de água, eletricidade e combustível de uso doméstico. A manutenção dos imóveis, móveis e eletrodomésticos e a garantia do fornecimento de água e energia, no caso de paralisação ou interrupção dos serviços, também é obrigação do Estado angolano (15)

Em 2015, o valor por médico cubano, independente da especialidade, era de US\$ 5.000, pagos mensalmente à Coorporação Antex S.A, por transferência bancária (15). Não há pagamento direto do país recrutador ao médico que presta o serviço. Segundo os entrevistados, o governo cubano fornece uma ajuda de custo para suprir despesas ao médico que está prestando serviço no exterior ao abrigo do acordo de cooperação. Não se teve acesso ao valor da ajuda de custo paga aos médicos cubanos que prestaram serviços em Angola.

A legislação angolana condiciona o exercício da medicina à inscrição do profissional no conselho de classe, apresentando quatro modelos de inscrição: i) médico angolano com licenciatura angolana; ii) médico angolano com licenciatura no exterior; iii) médico estrangeiro com residência; e iv) médico estrangeiro residente temporariamente em Angola por contrato (16). No caso das duas últimas situações, é exigido, pela Ordem dos Médicos, o certificado de reconhecimento ou equivalência do diploma ou certificado de licenciatura expedido pela Universidade Agostinho Neto (16).

No caso dos médicos cubanos que estão em Angola ao abrigo do acordo de cooperação, foi informado pelos entrevistados que estes submetem a documentação ao MINSA, que oferece ou não objeção. Não foram obtidas informações sobre a existência de 
processo de inscrição desses profissionais junto à Ordem dos Médicos em Angola. Dados do MINSA (13) atestaram 899 médicos cubanos atuando especialmente em hospitais públicos, nos serviços municipais de saúde, no combate aos vetores e na saúde mental.

Houve ampliação no número de atendimentos aos utentes com o recrutamento dos médicos cubanos. No período de 2010-2014, foram atendidos pelos profissionais cubanos uma média de 250 mil utentes angolanos e realizadas 7 mil cirurgias, entre eletivas e de urgência (13). Com relação às ações de atenção primária, segundo o MINSA, a cooperação com Cuba propiciou a melhoria no funcionamento e organização dos serviços, capacitação dos técnicos e melhoria nos indicadores de saúde pública. A assistência médica em unidades sanitárias de periferia e a realização de atividades por meio de equipes móveis garantiram assistência em saúde às populações de difícil acesso (13). No período de 20132015, foram realizados, pelas equipes móveis, atendimentos de saúde nas 18 províncias de Angola, notadamente nas aldeias rurais. No ano de 2013, 647 aldeias atendidas pelas equipes móveis; em 2014, 2.293 aldeias; e, em 2015, 472 aldeias (13). Em 2018 foram identificados 468 médicos cubanos, distribuídos em 38 especialidades, sendo 10 na área de medicina geral integral (MGI) (13).

A cooperação entre os dois países, no campo da saúde, também abrange o apoio a formação especializada, especialmente na abertura de novas escolas médicas em Angola, e na formação de internos nas especialidades médicas (17). O MINSA estima que no período de 2015-2019, 2.159 novos profissionais médicos estejam formados, apenas nas Faculdade de Medicina das províncias de Huambo, Cabinda, Huíla, Benguela e Malange, criadas a partir de $2008(13,17)$. Também foi relatado o envio de cerca de mil estudantes angolanos para formação em medicina em Cuba $(13,17)$.

\section{Cooperação médica Portugal-Cuba}

O Acordo de Cooperação Portugal-Cuba foi celebrado em 13 de junho de 2009, com o objetivo de contratar serviços médicos para prover cuidados de saúde, na área da atenção primária, para as zonas carenciadas, nomeadamente Algarve e Alentejo, e promover melhor acesso da população aos cuidados de saúde. Foram feitas renovações em 2010, 2011 e 2014 (18-21).

O Acordo estabeleceu requisitos mínimos para a recepção dos médicos cubanos pelo Sistema Nacional de Saúde de Portugal (SNS): médicos com especialidade em medicina geral e familiar (MGF), 10 anos de experiência profissional, cinco anos de experiência profissional no exterior e domínio da língua portuguesa. Em 2014, foi dispensado o requisito 
de experiência profissional no exterior e reduzido o tempo de experiência profissional para cinco anos (18-21). A modalidade eleita também foi a ATC; e o valor per capita por médico contratado era de 5.900 euros em 2009, sendo reduzido para 4.230 euros em 2014, data da última renovação. Esse valor era pago trimestralmente em conta bancária indicada pelos Serviços Médicos Cubanos (18-21). De acordo com as entrevistas realizadas com os médicos cubanos que prestaram serviços em Portugal ao abrigo do acordo, eles recebiam uma ajuda de custo no valor de 500 euros e, em Cuba, suas famílias recebiam o valor integral de sua remuneração referente aos serviços cubanos, como se lá estivessem trabalhando.

Sobre as obrigações assumidas pelo Estado português para execução durante a vigência do Acordo, tem-se: obrigação de fornecer alojamento; transporte e/ou ajuda de custo para o exercício das atividades quando esta for realizada em local afastado ou distinto da sede; assegurar pagamento de transporte aéreo durante o período de férias (ida-volta Cuba), e início e fim da missão; subsídio de alimentação; pagamento da anuidade referente à inscrição na Ordem dos Médicos. Aos médicos cubanos, foi garantido acesso ao SNS em condições de igualdade e condições aos nacionais (18-21). Na renovação de 2014 (21), foi inserida cláusula que determinava que os médicos cubanos só poderiam exercer atividades em Portugal enquanto vigente o acordo.

Segundo os entrevistados, para viabilizar a inscrição dos médicos cubanos na Ordem dos Médicos, o governo enviou à Cuba uma comissão, composta por representantes da Faculdade de Medicina da Universidade do Porto e da Ordem dos Médicos, que aplicaram testes de medicina redigidos em língua portuguesa, de modo a avaliar a capacidade clínica e o domínio da língua. Os candidatados considerados aptos tiveram a equivalência do seu diploma reconhecido, como exigido pela legislação vigente e, ao chegarem em Portugal, puderam se registrar na Ordem dos Médicos. Contudo, não foi reconhecida a especialidade de MGF exigida no acordo. Os médicos cubanos se registraram na condição de médicos indiferenciados (sem especialidade). Foi relatado ainda que houve, por parte do governo cubano, preparação para as provas, com a oferta de aulas de língua portuguesa e sobre as condições de saúde de Portugal. As aulas eras ministradas por especialistas cubanos. Os entrevistados relataram que, antes de início das atividades de campo, houve um rápido treinamento acerca dos programas de computadores necessários ao registro das atividades médicas a serem realizadas pelos médicos recrutados.

Os resultados demonstram que houve ampliação geográfica da área de atuação dos médicos cubanos em Portugal, atingindo quatro administrações regionais de saúde (ARS), de um total de cinco. Foram identificados médicos cubanos nas ARSs de a) Lisboa e Vale 
do Tejo; b) Alentejo; c) Centro e; d) Algarve. Portugal chegou a ter mais de 100 médicos cubanos atuando em seu território (22).

Avaliação conduzida pela Administração Central do Sistema de Saúde português (ACSS), em 2015, concluiu pelo aspecto positivo gerado com a cooperação, relatando aumento da acessibilidade; redução do número de utentes sem médico da família e aumento de iniciativas de programas de promoção à saúde (23).

\section{Cooperação médica Brasil-Cuba}

Em 2013 o Estado brasileiro recrutou médicos cubanos para preencher vagas ociosas do projeto voltado ao provimento emergencial de médicos para áreas prioritárias do SUS, intitulado Projeto Mais Médicos para o Brasil (PMMB) (24). Os cubanos são chamados de médicos cooperados, porque são recrutados mediante cooperação técnica estabelecida entre Brasil e Cuba, com a intermediação da Organização Pan-Americana da Saúde (OPAS/OMS) (24).

Os critérios estabelecidos exigiam que o médico cubano fosse especialista em medicina geral integral, possuísse 10 anos de experiência profissional, e mínimo de dois anos de trabalho em outro país. Também foi exigido o conhecimento básico da língua portuguesa. Os médicos cubanos poderiam ser alocados em qualquer das áreas prioritárias previamente indicadas, que atendessem a condições específicas estabelecidas pelo PMMB: i) municípios com $20 \%$ ou mais da população, vivendo em condição de alta vulnerabilidade social; ii) periferias das capitais e regiões metropolitanas; iii) municípios com mais de 80 mil habitantes, que apresentam os mais baixos níveis de receita pública per capita do país; e iv) 25 Distritos Sanitários Especiais Indígenas (DSEI) (25).

O PMMB foi concebido na modalidade de ensino-serviço, sendo o médico recrutado acompanhado por um médico supervisor e um tutor acadêmico. Antes de iniciarem as atividades de campo em suas localidades, os médicos passaram por um processo de formação, com duração de quatro semanas. Foram ministrados conteúdos sobre legislação do Sistema Único de Saúde brasileiro (SUS); funcionamento e atribuições do SUS, especialmente AP; protocolos clínicos de atendimentos definidos pelo Ministério da Saúde; língua portuguesa; e código de ética médica. Ao final de um ano, e completado o percurso formativo, o médico recebe um título de especialização lato sensu (26).

O pagamento pelos médicos cubanos recrutados é feito pelo governo brasileiro à OPAS, não havendo relação direta entre Brasil e Cuba. O cálculo per capita é feito com base no valor mensal da bolsa formação, paga indistintamente a todos os médicos que participam 
do PMMB. Em 2016 o valor mensal dessa bolsa era de R\$10.570,88. Segundo os médicos cubanos entrevistados no Brasil, o governo cubano repassava ao médico o valor de US\$ 1.100 a título de ajuda de custo; e suas famílias recebiam o salário devido em Cuba, como se lá estivessem em atividade.

Com relação às obrigações assumidas com a assinatura do Termo de Cooperação Técnica entre Brasil e OPAS/OMS, foram identificados 12 termos de ajustes no período 2013-2018, com o estabelecimento de obrigações para Brasil e OPAS/OMS. Coube ao Brasil, garantir os documentos migratórios e demais necessários a permanência do médico durante o Programa; garantir aperfeiçoamento por meio de curso de especialização; transporte internacional dos médicos participantes, inclusive por ocasião de gozo do recesso; deslocamento dos médicos cubanos no território nacional; recesso de 30 dias sem prejuízo do recebimento da bolsa; garantia de atenção à saúde e segurança; entre outras. Coube à OPAS/OMS: avaliar a seleção dos médicos aptos a participar do PMMB, observando os seguintes critérios: médico com diploma expedido por instituição de educação superior; habilitação para o exercício da medicina em país com relação estatística médico-habitante igual ou superior a 1,8/1.000; declaração de regularidade no conselho de classe do país onde o médico estiver habilitado para exercer a medicina; declaração de conhecimento da língua portuguesa; declaração de nada consta na esfera criminal, emitida pelo país em que está habilitado para o exercício da medicina; entre outros.

Com relação à inscrição do médico cubano no conselho de classe, a legislação que instituiu o Programa Mais Médicos dispensou a necessidade do registro prévio do diploma e da inscrição no conselho profissional (25). O Ministério da Saúde, por meio da coordenação do Projeto, emitia um Registro Único do Ministério da Saúde (RMS) e respectiva carteira de identificação para os médicos atuantes no PMMB, que os habilita para o exercício da medicina exclusivamente no âmbito do Projeto. Os médicos, contudo, ficavam sujeitos à fiscalização dos conselhos de medicina.

Durante o período de 2013-2018, mais de 13 mil médicos cubanos atuaram no Brasil, ao abrigo do Acordo (26). Inúmeros estudos foram produzidos acerca do PMM, relatando os benefícios advindo da cooperação, especialmente no que diz respeito à ampliação do acesso às ações e serviços de saúde; fortalecimento da APS; redução de desigualdades em saúde $(17,27-30)$. Destaca-se a presença de médicos cubanos em todos os distritos sanitários indígenas (26). 


\section{Discussão}

Da análise, percebe-se que as estratégias utilizadas pelos países envolvidos na cooperação médica por vezes se aproximam, mas contextos específicos conduzem à busca por soluções peculiares, que se harmonizem com a legislação vigente em cada país. Segundo Bucci e Coutinho (31) essas estratégias são, na verdade um esforço intencional voltado à realização do objetivo desejado, traduzido em um componente jurídico que se insere na dinâmica e complexa engrenagem desse agir estatal.

Esse papel pode ser vislumbrado especificamente ao analisar os casos de Portugal e Angola, que elegeram firmar acordo de cooperação diretamente com Cuba, enquanto o Brasil optou por estabelecer cooperação técnica com a OPAS/OMS, para o atingimento dos objetivos pactuados, dada as dimensões da contratação, que mobilizaram quantidade considerável de recursos humanos e financeiros.

Molina, Tasca e Suárez (32), ao analisarem a cooperação firmada entre OPAS/OMS e Brasil para o PMM, destacam o caráter inovador e a complexidade do processo de recrutamento, preparação e coordenação das ações. Destacam ainda a sinergia entre os atores; o compartilhamento de responsabilidades em determinados momentos e a responsabilidade quase que integral da OPAS/OMS em outros momentos, como no caso da mobilização e alocação dos médicos; e a administração dos recursos financeiros. Outros autores, na mesma linha, reconhecem a triangulação Brasil-OPAS/OMS-Cuba como inovadora no aspecto da cooperação internacional e fundamental para o sucesso da iniciativa $(17,26,33)$.

O modelo de cooperação eleito pelos países recrutadores foi distinto. Portugal e Angola optaram pela dimensão da prestação de serviços médicos, nos moldes da assistência técnica compensada (14). O Brasil, por sua vez, elegeu a dimensão ensino-serviço, propiciando aos médicos cubanos que ingressaram no PMMB um processo de formação aliado à prestação de serviços, permitindo um maior ganho e compartilhamento de experiências $(17,26)$.

Em uma análise alargada, é possível verificar que as obrigações e compromissos insertos nos termos de cooperação também estão diretamente relacionados à governança das ações políticas.

Em que pese não ser objeto da presente análise, não se pode olvidar que o estudo demonstrou ser possível realizar recrutamentos de médicos de maneira não predatória, na medida em que os países recrutadores observem a densidade médico/habitante nos países 
com RHS recrutados, atendendo assim a recomendação do Código Global de Práticas para o Recrutamento Internacional de Pessoal de Saúde (34).

Sobre o tema do exercício da medicina pelos médicos estrangeiros, apesar dos países investigados possuírem legislação específica sobre validação e reconhecimento de cursos de medicina realizados no exterior, os três países optaram pela adoção de medidas específicas - mais ou menos flexíveis - que facilitaram esse processo. O Brasil optou por alterar seu quadro normativo-legal, instituindo uma exceção ao processo de revalidação do diploma aplicada exclusivamente aos médicos estrangeiros que atuavam no PMMB (25). menor número de médicos recrutados por Portugal permitiu ação distinta, que, além de não implicar em alteração legislativa, demonstrou habilidade política do governo em negociar com as instituições responsáveis por esse tema. Ausência de dados sobre Angola prejudicou a análise desse contexto.

Ponto que se revelou bastante polêmico no estudo foi o pagamento aos médicos cubanos, já que não houve recebimento direto do salário/ bolsa pelos serviços prestados, e sim uma ajuda de custo. Nesse aspecto, importante considerar que os médicos cubanos, ao atuarem em missões internacionais, têm apoio estruturado do governo cubano durante o período que estão no exterior; continuam a receber o salário regular do governo cubano, acrescido de um valor mensal por estarem em missão; e, ao retornarem, têm possibilidade de ascensão e progressão na carreira (35).

Os benefícios gerados são perceptíveis não apenas na prestação de cuidados de qualidade e ampliação do acesso, amplamente documentado em artigos e relatórios técnicos $(3,4,5,17,22,23,26,27-30)$, mas também no desenvolvimento de uma expertise voltada às ações de governança no campo político, de educação e gestão de recursos humanos, fortalecendo o papel dos ministérios da saúde.

Para Cuba, o internacionalismo médico representa benefícios financeiros e políticos diretos e/ou ganhos em acordos bilaterais (35).

\section{Considerações finais}

O recrutamento de RHS continua sendo importante ação em busca da ampliação do acesso às ações e serviços de saúde. Contudo, o êxito dessas iniciativas depende não apenas da quantidade e qualidade de RHS empregados, mas de um processo articulado de cooperação, que envolva a capacidade institucional e política de superar as barreiras de implementação da estratégia eleita. 


\section{Referências}

1. Dussault G, Dubois CA. Human resources for health policies: a critical component in health policies. Hum Resour Health. 2003;1(1).

2. Buykx P, Humphreys J, Wakerman J, Pashen D. Systematic review of effective retention incentives for health workers in rural and remote areas: towards evidence-based policy. Aust J Rural Health. 2010; 18(3):102-9.

3. Dussault G, Franceschini MC. Not enough there, too many here: understanding geographical imbalances in the distribution of the health workforce. Hum Resour Health. 2006; 4:12. [citado em 16 mai 2015].

4. World Health Organization. World health statistics 2006 [internet]. Geneva: WHO; 2006;

5. Cuba. Unidad Central de Cooperación Médica. Anuário 2017. La Habana; 2017.

6. Osa JA. Um olhar para a saúde pública cubana. Estud av. 2011; (25)72:89-96.

7. Cárdenas LG. La especialidad de medicina familiar en la formación de los recursos humanos en Cuba. Mundo saúde. 2010; (34)3:404-6.

8. Biernacki $P$, Waldorf $D$. Snowball sampling: problem and techniques of chain referral sampling. Sociol Methods Res.1981; 10:141-63.

9. Handcock MS, Gile KJ. On the concept of snowball sampling. Sociological Methodology. Aug 2011;41(1).

10. United Nations Development Programme. Human Development Indices and Indicators: 2018 Statistical Update. Nova York: UNDP; 2018.

11. Index Mundi. Comparação entre países: produto interno bruto (PIB).

12. World Health Organization. World health statistics 2018: monitoring health for the SDGs, sustainable development goals. Geneva: WHO; 2017.

13. Ministério da Saúde (Angola). Memorando de apoio à Visita de Sua Excelência Senhor Ministro da Saúde a Cuba (20-24 abr 2015). Angola; 2015. 24p.

14. Ramalho Filho JE, Lima AEI. O Programa Mais Médicos e a cooperação trilateral Brasil-OPAS-Cuba para o fortalecimento da atenção básica no SUS. Brasil. In: Brasil. Ministério da Saúde. Assessoria de Assuntos Internacionais de Saúde. Saúde e Política Externa: os 20 anos da Assessoria de Assuntos Internacionais de Saúde (1998-2018). Brasília: Ministério da Saúde; 2018.

15. Angola e Cuba. Contrato de Prestación de Servicio de Asistencia Técnica Extranjera em ele sector de la salud. Angola; 2015.

16. Ordem dos Médicos de Angola. Estatuto . [citado em 10 mai 2016]. 
17. Alves SMC, Oliveira FP, Matos MFM, Santos LMP, Delduque MC. Cooperação internacional e escassez de médicos: análise da interação entre Brasil, Angola e Cuba. Ciên Saúde Colet. 2017;22(7):2223-35.

18. Acordo de cooperação para a prestação de serviços médicos entre Serviços Médicos Cubanos da República de Cuba e Administração Central dos Sistemas de Saúde do Ministério da Saúde da República Portuguesa; 2009.

19. Il Renovação do acordo de cooperação para a prestação de serviços médicos entre Serviços Médicos Cubanos da República de Cuba e Administração Central do Sistema de Saúde do Ministério da Saúde da República Portuguesa; 2010.

20. III Renovação do acordo de cooperação para a prestação de serviços médicos entre Serviços Médicos Cubanos da República de Cuba e Administração Central do Sistema de Saúde. IP do Ministério da Saúde da República Portuguesa; 2011.

21. IV Renovação do acordo de cooperação para a prestação de serviços médicos entre Serviços Médicos Cubanos da República de Cuba e Administração Central do Sistema de Saúde. IP do Ministério da Saúde da República Portuguesa; 2014.

22. Serviço Nacional de Saúde. Relatório Social do Ministério da Saúde e do Serviço Nacional de Saúde. Lisboa: SNS; 2017.

23. Portugal. Administração Central do Sistema de Saúde. Avaliação dos médicos cubanos. IV Renovação do Acordo de Cooperação para a prestação de serviços médicos entre Serviços Médicos Cubanos da República de Cuba e ACSS, IP. do Ministério da Saúde da República Portuguesa. Lisboa: ACSS; 2015.

24. $3^{\circ}$ Termo de Ajuste ao 80 Termo de Cooperação Técnica para o desenvolvimento de ações vinculadas ao projeto Ampliação do aceso da população brasileira à atenção básica em saúde, que entre si celebram o Ministério da Saúde/Fundo Nacional de Saúde e a Organização Pan-Americana da Saúde/Organização Mundial da Saúde. 2013.

25. Brasil. Lei $n^{\circ} 12.871$, de 22 de Outubro de 2013. Institui o Programa Mais Médicos, altera as Leis $n^{\circ} 8.745$, de 9 de dezembro de 1993, e $n^{\circ} 6.932$, de 7 de julho de 1981 e dá outras providências. Diário Oficial da União 23 out 2013; Seção 1:1-4.

26. Ministério da Saúde. Programa Mais Médicos - dois anos: mais saúde para os brasileiros. Brasília: Ministério da Saúde; 2015.

27. Comes Y, Trindade JS, Shimizu HE, Hamann EM, Bargioni F, Ramirez L, Sanchez MN, Santos LMP. Avaliação da satisfação dos usuários e da responsividade dos serviços em municípios inscritos no Programa Mais Médicos. Ciênc. saúde coletiva. 2016; 21(9): 27492759.

28. Girardi SN, Stralen ACS, Cella JN, Maas LWD, Carvalho CL, Faria EO. Impacto do Programa Mais Médicos na redução da escassez de médicos em Atenção Primária à Saúde. Ciênc. saúde coletiva. 2016; 21(9): 2675-2684. 
29. Lima RTS, Fernandes TG, Balieiro AAS, Costa FS, Schramm JMA, Schweickardt JC, Ferla AA. A Atenção Básica no Brasil e o Programa Mais Médicos: uma análise de indicadores de produção. Ciênc. saúde coletiva. 2016; 21(9): 2685-2696.

30. Santos LMP, Oliveira A, Trindade JS, Barreto ICHC, Palmeira PA, Comes Y, Santos FOS, Santos W, Oliveira JPA, Pessoa VM, Shimizu, HE. Implementation research: towards universal health coverage with more doctors in Brazil. Bull World Health Organ. 2017; 95(2): 103-112.

31. Bucci MPD, Coutinho DR. Arranjos jurídico-institucionais da política de inovação tecnológica: uma análise baseada na abordagem de direito e políticas públicas. In: Coutinho DR, Foss MC, Mouallem PSB, organizadores. Inovação no Brasil: avanços e desafios jurídicos e institucionais. São Paulo: Blucher; 2017. p.313-39.

32. Molina J, Tasca R, Suárez J. Monitoramento e avaliação do Projeto de Cooperação da OPAS/OMS com o Programa Mais Médicos: reflexões a meio caminho. Cien Saude Colet. 2016; 21(9):2925-2933.

33. González JEC, Morales ECL, Ramos AD. Proyecto Mais Médicos para Brasil: nueva modalidad de colaboración cubana en el exterior. Rev Edumecentro. 2015; 7(1):171-177.

34. Sixty-Third World Health Assembly, Agenda Item 11.5, WHO Global Code of Practice on the International Recruitment of Health Personnel, WHA63.16 (May 21, 2010).

35. Huish R. Why Does Cuba 'Care' So Much? Understanding the Epistemology of Solidarity in Global Health Outreach. Public Health Ethics. 2014; 7(3):261-276

\section{Colaboradores}

Alves SMC contribuiu com o desenho do estudo, coleta e análise dos dados e redação do artigo. Delduque MC e de Faria PL contribuíram com o desenho do estudo e aprovação da versão final do artigo.

\section{Como citar este artigo}

Alves SMC, Delduque MC, de Faria PL. Recrutamento de médicos cubanos: análise comparativa da estratégia adotada por Angola, Portugal e Brasil para compor seus recursos humanos em saúde. Cadernos Ibero-Americanos de Direito Sanitário. 2021 dez.;10(Suplemento):71-85. 